\title{
After the goldrush
}

"I dreamed, I saw the silver spaceships, flyin' in the yellow haze of the sun"

Neil Young (after the goldrush)

Daß der x-te Aufguß des guten, alten Songs aus den Siebzigern, digitally remastert auf CDROM, Absatzzahlen bringt, die Lichtjahre über der bestverkauften juristischen Fach-CDROM liegen - wer wollte dieser Schätzung widersprechen?

Nix war's mit dem Traum der Verlage vom weltraumgreifenden Höhenflug der silbernen Scheiben in den goldenen Sonnenschleier des Profits. Die Positionierung des neuen Mediums am Markt als juristisches Arbeitsmittel der Zukunft ist im ersten Anlauf fehlgeschlagen. Die Anbieter mußten lernen, daß beim allergrößten Teil der Juristen vielleicht noch auf längere Zeit das Verständnis, die Arbeitsweise, der gute Wille oder einfach die Hardware fehlen, die dem Medium CD-ROM zum durchgreifenden Erfolg verhelfen könnten. Welcher Anwalt begreift schon einen Computer als Informationsmittel und nicht als Schreibgerät, mit dessen Beherrschung sich allenfalls seine Sekretärin zu befassen hat?

Wie bei Träumen üblich, erinnert man sich ihrer post noctem nicht oder nur schemenhaft. Man meint noch vage zu erkennen, daß man in klarer Risikosicht nur den Notwendigkeiten gefolgt ist, um am Ball zu bleiben, Know-How zu schaffen, vorsichtige Grundschritte in die Zukunft zu lenken ...

Die teilweise immensen Entwicklungskosten, die von den juristischen Verlagen in die neuen Produkte investiert wurden, sprechen dem darwider. Hier hat man sich mehr erwartet. Die Branche, in der mittleren Linie meist gut gepolstert, steckt das zähneknirschend weg, schreibt ab. Aber eine gewisse Agonie macht sich schon breit: Allzu lang lassen lang angekündigte Produkte auf sich warten, große Neuheiten fehlen und auch das Schrumpfen manch elektronischen Lektorats läßt leicht schließen, daß die Kaufleute in den Verlagen den Geldhahn zudrehen.

Wenn die erste Garnitur der Goldgräber ermattet, drängen die Mitläufer auf den Claim und wühlen den Sand nochmal durch. Insbesondere Gesetzestexte sind glänzende Körnlein, die jeder heben und auf die Scheibe pressen kann. Wundersam, wer so was alles produziert, ohne Gewähr für Herkunft und die unabdingbare Richtigkeit und Aktualität. Scanner ersetzt Redaktion - gottlob fördern auch diese Schöpfungen wohl nur Katzengold zutage.

Wie bei jedem Goldrausch wurde allerdings auch mancher ansehnliche Brocken gehoben, der mittlerweile noch kräftig bearbeitet und poliert wurde. Auch wenn es sich oft noch nicht rentiert: der Einsatz der Verlage hat heute zu einer Vielzahl beachtlicher und hochwertiger elektronischer Produkte für Juristen geführt. Trotz aller Schwierigkeiten gibt es neue Ideen - sie und eine moderatere Preispolitik könnten die Wende zu einer breiteren Akzeptanz schaffen. Eine Übersicht erhalten Sie in diesem Digest.

Wenn nach erster Euphorie und der Angst, zu spät zu kommen, der Blick auf ein mähliches Fortschreiten gerichtet wird, das mit der Entwicklung des Informations-, Arbeits- und Kaufverhaltens bei den ins Auge gefaßten Zielgruppen parallel einhergeht, dann stimmt eines Tages sicher auch die Bilanz.

Bevor wir uns aber hiervon eine zarte Morgenröte an den Horizont malen lassen, wenden wir uns doch lieber in die Richtung, aus der uns die neueste Supernova blendend ins Hirn schießt: Das ganze juristische Wissen, online durchsurft und downgeloaded aus Interjur und Compujus - das wird ein Geschäft!

"All in a dream, all in a dream, the loading had begun"

Neil Young (after the goldrush)

Nürtingen-Hardt, den 22. September 1995

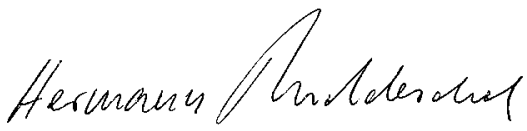

(Hermann Ruckdeschel) 